\title{
Lifespan Enhancement of Wireless Sensor Network (WSN) through Residual Energy Balancing and Uniform Distribution of Cluster Nodes
}

\author{
Mohanaradhya ${ }^{1}$ and K.A. Sumithra Devi ${ }^{2}$ \\ 'Department of MCA, R.V College of Engineering, Bengaluru-560059, Karnataka, India \\ Mohanaradhya72@gmail.com \\ 2GSSS Institute of Technology, Mysore-570016, Karnataka, India. sumithraka@gmail.com
}

\begin{abstract}
In the era of computing technology WSN is playing significant role in human life by helping environment monitoring, health monitoring, habitat monitoring and military applications. In cluster based WSN network energy can utilized optimally to enhance the lifetime by uniform distribution of nodes among the clusters and avoiding redundant data transmission from sensing nodes to sink through balanced utilization of network energy. In dense WSN, nodes in the same sensing range collects the same data and hence it is not required for all the nodes to transmit the data to the Cluster Head. This paper proposes a method which uniformly attaches the nodes to Cluster Heads and avoids the duplicate data transmission from different nodes in the same sensing range thereby conserving energy. The proposed method distributes the nodes uniformly and checks the residual energy among the nodes having the same data to allow the node with more residual energy to transmit the data to the cluster head. This results in increase of $3 \%$ alive nodes compared to earlier method5, leading to an increased lifespan.
\end{abstract}

Keywords: Energy Balancing, Redundant Data, Residual Energy, Threshold Distance, Uniform distribution, WSN.

\section{Introduction}

In this computer era, the processing devices are collaborated with other inter disciplinary devices like sensors and transceivers to create independent nodes with sensing, processing and transceiver capacity. Collection of such nodes for the betterment of human life, constructs Wireless Sensor Network. Wireless Sensor Networks (WSN) improved human life by helping in military, environment, health care, home and industrial applications. In (WSN) each node is multi-functional and consumes energy from the battery unit attached. The life span of the network is dependent on the power unit with limited capacity.

The energy drain out in a node not only affects itself but also may lead to expire of a part of a network. Node energy can be stringently spent by forwarding the packets only through the dense energy area so that nodes with low residual energy may be rescued to increase operational life span of WSN. Every node loses $80 \%$ of its energy for transmitting datal. The routing protocol tries to conserve energy by efficient routing ${ }^{2}$. The energy of the network can be conserved by reducing the number of data packets transmitted between the nodes. In data collection wireless sensor networks, as the nodes are densely deployed, the data collected are normally redundant and similar. If every node sends data to the sink, network energy will drain out quickly by wasting energy in transmitting redundant data and also the energy is wasted in aggregation at cluster head as the data size increases due to redundant data received ${ }^{3}$. Previous research ${ }^{1,5}$ shows that the duplicate data at Cluster Head $(\mathrm{CH})$ can be reduced by denying redundant data transmission from cluster members to $\mathrm{CH}$, based on the residual energy of the cluster member and sensing range. These methodst, expands the life span of network by allowing the node with more

*Author for correspondence 
residual energy to transmit for balanced utilization of node energy. Many energy efficient methods--9 proposed techniques to balance the energy consumption among the $\mathrm{CH}$ so as to extend the life span of WSN. Generally $\mathrm{CH}$ extracts more energy as it consumes energy for summarizing data, receiving data from multiple nodes and for transmitting to the base station. In DBCHS cluster heads are distributed evenly and only minimal nodes are selected as $\mathrm{CH}$ across the network to stretch the lifetime. This paper proposes a method to attach uniform nodes to $\mathrm{CH}$ and avoids the redundant data transmission between the normal nodes and cluster head so that both the transmission and aggregation energy can be conserved. The life span of the network is extended by balanced spending of node energy..

\section{Related Work}

This section gives the analysis and understanding the mechanisms for cluster head selections, the different scenarios of redundant data, and the use of residual energy criteria. With these issues, this paper proposes a method to associate uniform number of nodes to $\mathrm{CH}$ and to reduce the redundant data transmission between the sensing node and the cluster head by considering the node residual energy as criteria. So that the node residual energy utilization is balanced among all the nodes. Redundant data are avoided and prolongs the network lifespan.

\subsection{Low- Energy Adaptive Clustering Hierarchy (LEACH)}

It is a self-organizing and adaptive clustering protocol proposed by Heinzelman. The operation of LEACH is parted into rounds, where every round begins with a setup phase for cluster formation, followed by a steadystate phase; when data is conveyed to the sink node occur through Time Division Multiplexing Approach (TDMA). LEACH protocol uses the random opting of cluster heads to achieve load balancing between the sensor nodes selected as $\mathrm{CH}$. LEACH has some issues.

- In LEACH, a sensor node is elected as the cluster head, according to a distributed probabilistic approach. The sensor node gets associated with the cluster head to form a cluster, based on the signal strength i.e., by considering the distance between the cluster head and the sensor node. Nodes join the cluster head, which is nearer to them. Once the clusters are formed, cluster heads allocates the time slot to transmit the data. This approach insures lower message overhead, but cannot ensure that cluster heads are distributed over the entire network uniformly as there is a probability for nearby nodes to become cluster head and also the nodes near the sink get selected as $\mathrm{CH}$. Both cases lead to choosing of unnecessary $\mathrm{CH}$.

- Uneven distribution of the cluster heads in the absorption area leads to load imbalance over the cluster heads and unnecessary investment of energy by cluster heads. To achieve uniform distribution of $\mathrm{CH} \mathrm{DBCHS}^{10}$ considers distance between the nodes as one of the criteria to select $\mathrm{CH}$.

The above two issues were addressed by us in Distanced based cluster head section for WSN.

\subsection{QIBEEC (Quality of Information Based Energy Efficient Clustering) algorithm.}

In the network where the nodes are densely positioned, the probability of transmitting redundant data to the $\mathrm{CH}$ is more. This leads to waste of energy at sending nodes due to redundant data transmission and data aggregation energy at the cluster heads.

Manjuprad suggested a method to avoid redundant data transmission. This algorithm operates in three phases, cluster head selection phase, formation of cluster and Transmission phase with redundant data avoidance. In the first phase the cluster heads are opted by considering the criteria threshold energy and threshold distance. The threshold energy is given by the following equation.

Threshold distance is calculated as

$$
\begin{aligned}
& T_{d}=\sqrt{N_{d^{2}}+N_{d^{2}}} \\
& N_{d}=\frac{A}{4 L}
\end{aligned}
$$

Where $\mathrm{A}=$ Sensing area in square, $\mathrm{L}=$ Length of sensing area, $E_{k}=$ Transmission Energy, $E_{d a}=$ Data aggregation energy. $k$ =packet length in bits. Nodes satisfying the threshold energy and distance will be opted as cluster head. Cluster formation phase operates as in LEACH. In the transmission phase, redundant data is identified by checking whether it is the same sensing range of other active node, if so it goes to sleep mode. The 
nodes decides that the data in it is redundant or not based on the threshold range

$T_{(r)}=\frac{S_{r}}{N}(L)$

Where $S_{r}=$ Sensing range of node, N=Number of nodes, L=Length of sensing area. QIBEEC algorithm tries to extend network life time by avoiding redundant data from nodes to $\mathrm{CH}$. It considers residual energy in selecting the $\mathrm{CH}$, but this method is not taking node residual energy into account to avoid redundant data transmission. Distributed, uniform consumption and appropriate management of cluster member residual energy extents the network lifespan.

\subsection{WSN Lifetime Enhancement through Residual energy balancing by Avoiding Redundant Data Transmission (LERBARDT).}

Mohanaradhya, Sumithradevi.K proposed a method that checks the residual energy among the nodes having the same data and allows the node with more residual energy to transmit the data to the cluster head. This leads to balanced and distributed utilization of node energy thereby the network lifetime is enhanced. This method maintains a threshold distance between the cluster heads to distribute the $\mathrm{CH}$ uniformly in network area and also to avoid the nodes within the threshold distance from sink to become cluster head. This algorithm calculates threshold distance based on the minimum transmitting range of the deployed nodes which varies depending on type of node. The threshold distance $r$ is calculated as

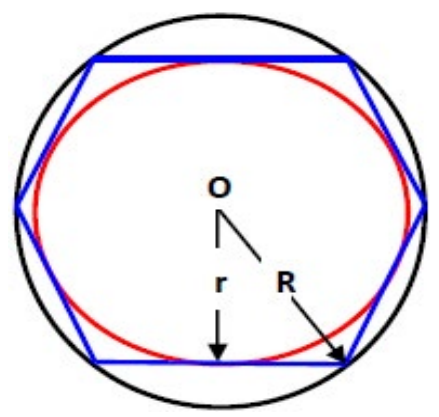

Figure 1. Threshold transmitting distance.

$$
r=\frac{1}{2 * R \sqrt{3}}
$$

In figure $1 \mathrm{O}$ shows the node position, $\mathbf{r}$ is the threshold distance and $\mathrm{R}$ shows the minimum transmitting range of a node. Once the threshold distance is calculated, it selects No Clusterhead Region (NCR). This is the network area around the sink covered by radius $\mathbf{r}$ In cluster head selection step one node outside this NCR region is randomly opted as cluster head, the next node eligible for cluster head will check whether it is in the threshold distance from other cluster head. If not within the distance $\mathbf{r}$ then node will become cluster head otherwise it will join as member node. In data transmission phase redundant data is avoided by allowing only the node with more residual energy to transmit data to sink. Redundant data is identified based on threshold sensing range $T_{r}$ calculated as in Nodes within the same threshold sensing range will have the same data and node with more residual energy will be permitted to transmit data to the sink.

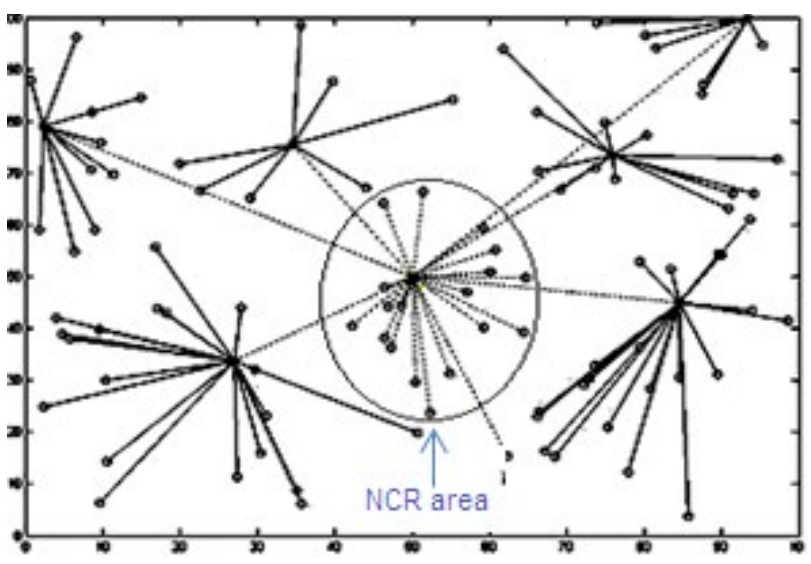

Figure 2. Cluster Head selection

In Figure 2 the center region around the sink shows No Cluster Head Region (NCR) and it is observed that the cluster heads are uniformly distributed in the network region by avoiding the selection of unnecessary cluster heads. So this method distributes $\mathrm{CH}$ uniformly and avoids redundant data to improve life time, but this method is not looking at the uniform distribution of member nodes among the clusters. In Figure 2 it is observed that each cluster head is attached with unequal number of nodes, by equal distribution, residual energy consumption among the clusters can be stabilized. 


\section{Proposed Work}

The proposed method tries to balance the distribution of member nodes among the clusters formed in each round. The proposed method works in five steps.

Step1: Calculates the threshold transmitting distance based on the minimum transmission range of the nodes deployed in the network as in theshold distance between the nodes is considered as the criteria to select the Cluster head.

Step2: Based on the calculated threshold transmission range, No Cluster head Region (NCR) is selected with respect to threshold distance from sink. Nodes within this NCR area can directly send data to the sink.

Step3: Step three is cluster head selection phase, one node from outside the NCR region is randomly selected as cluster head. The next eligible node, will check either it is within the threshold distance of any already selected cluster head. If beyond the threshold distance, node will act as cluster head otherwise it will join as the cluster member.

Step4: In this step selected cluster heads will advertise their cluster head status message to other nodes in the network. Each node determines to which cluster head it should get associate with, based on the signal strength received. This step restricts the number of nodes getting associated with each cluster head to attach equal number of nodes to each cluster head by applying

$\mathrm{N}_{\mathrm{sn}}=$ Number of sensing nodes restricted to each cluster.

$\mathrm{N}_{\mathrm{an}}=$ Number of alive nodes. $\mathrm{N}_{\mathrm{ncr}}=$ Number of alive nodes in NCR area.

$\mathrm{N}_{\mathrm{CH}}=$ Number of Cluster Heads in each round.

Once the $\mathrm{N}_{\mathrm{sn}}$ number of nodes attached to cluster head, sensor node get attaches to next nearest cluster head.

Step5: After forming the cluster each node gets TDMA slot for transmitting data to cluster head. When a node gets time slot, it checks for the redundant data based on the threshold sensing range as calculated inf, assuming that nodes in the same sensing range will have the same data. Nodes in the same sensing range will exchange their residual energy information. The node with less residual energy will be idled. Uniform distribution of sensors in clusters and permitting of sensor with higher residual energy to transmit, balances the consumption of energy in every cluster and also balances the dead nodes in each cluster, so that it increases the operational time of the network.

\section{Results and Discussion}

Performance of LEREBUDN is assessed by considering the number of dead nodes and number of packets transmitted to the cluster head. Simulation is done with network of 200 nodes having initial energy $0.05 \mathrm{~J}$ in each node. The algorithm is simulated for 200 rounds in the network area of $100 \times 100 \mathrm{~m}$ with $25 \mathrm{~m}$ and $5 \mathrm{~m}$ as transmitting and sensing range respectively. It is assumed that all nodes are homogeneous and sink is positioned at center.

The observed values are compared with Distance Based Cluster Head Section (DBCHS) in sensor networks for Efficient Energy Utilization If WSN Lifetime Enhancement through Residual energy balancing by Avoiding Redundant Data Transmission (LERBARDT) and LEACHP.

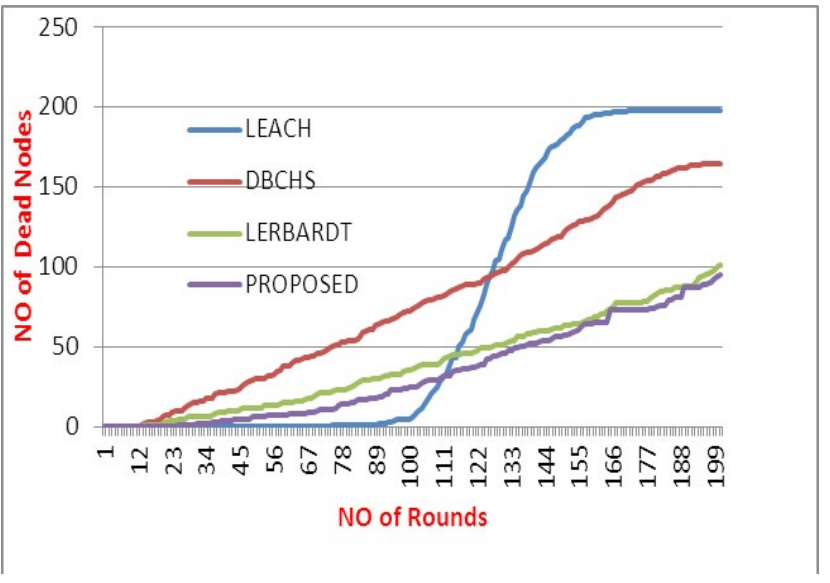

Figure 3. Number of failure nodes in each round.

From the Figure 3 it may be concluded that proposed one is energy efficient than LEACH, DBCHS and LERBADT as the number of inactive nodes are 200,164, 101 and 95 respectively on the last round. In proposed method there are $3 \%$ more alive nodes compare to the previous method LERBARDT and it is also observed that during initial rounds nodes death rate is slow.

\section{Conclusion and Future Work}

Forming clusters with uniform number of sensor nodes stabilizes death speed of nodes and permitting nodes with 
more residual energy to transmit data avoids redundant data between nodes and $\mathrm{CH}$. By this the lifespan of the sensor network is extended.

\section{References}

1. Nath GN, Thimmaiah HS, Kawale SR, Ramya, Priya EB. Enhancing Energy Efficiency in WSN using Energy Potential Concepts.International Conference on Advances in Computer and Electrical Engineering (ICACEE'2012). Manila, Philippines: 2012 Nov. 17-18.

2. Akyildiz IF, Vuran MC. Wireless Sensor Networks. Wiley Publications; 2010.

3. Doomun R.READA: Redundancy Elimination of Accurate Data Aggregation in WSN. 2011 April; 300-8. DOI:10.4236/ wsn.2010.24041.

4. Prasad M, Dharani A. A qoi based energy efficient clustering for dense wireless sensor network. International Journal Of Advanced Smart Sensor Network Systems (IJASSN). 2013 Apr; 3(2):.

5. Mohanaradhya, Sumithra Devi KA.WSN Lifetime Enhancement through Residual energy balancing by Avoiding Redundant Data Transmission. $6^{\text {th }}$ International Conference on Advances in Computing, Control \& Telecommunication Technologies (ACT2015): De Gru yter, 2016. 73-83. DOI:10.1515/9783110450101-0096.

6. Kimura N,Latifi S. A Survey on Data Compression in Wireless Sensor Networks.Proceedings of the International Conference on Information Technology: Coding and Computing (ITCC'05). IEEE; 2005.

7. Lee S, Choe H, Park B, Song Y, Kim C-K. LUCA: An energy-efficient unequal clustering algorithm using location information for wireless sensor networks. Wireless Personal Communications. 2011;56( 4):715-31.
8. Lee S, Lee J, Sin H, Yoo S, Lee Y, Kim S. An energy-efficient distributed unequal clustering protocol for wireless sensornetworks. World Academy of Science, Engineering and Technology. 2008;48:443-7.

9. Heinzelman WR, Chandrakasan A, Balakrishnan H. Energy efficient communication protocol for wireless micro networks. Proceedings of the 33rd Annual Hawaii International Conference on System Sciences (HICSS), Maui, Hawaii. 2000 Jan. 3005-14.

10. Mohanaradhya, Sumithra Devi KA, Dharani A. Distance based cluster head section in sensor networks for efficient energy utilization. International Journal of Advanced Research in Engineering and Technology (IJARET). 2013 Jan; 4(1).

11. Padmavathy TV,, Chitra M. ,Extending the Network Lifetime of Wireless Sensor Networks Using Residual Energy Extraction -Hybrid Scheduling Algorithm.International Journal of Communications, Network and System Sciences. 2010 Jan; 3: 98-106.

12. Kole S, Vhatkar KN, Bag VV. Distance Based Cluster Formation Technique for LEACH Protocol in Wireless Sensor Network. International Journal of Application or Innovation in Engineering \& Management (IJAIEM). 2014 Maarch; 3(3):334-8.

13. Chen G,Chengfa Li, Mao Ye,Jie Wu. An unequal clusterbased routing protocol in wireless sensor networks. Wireless Networks. 2009 Feb; 15(2);193-207.

14. Anastasi G, Conti M, Francesco MD, Passarella A. Energy conservation in wireless sensor networks: A survey. Ad Hoc Networks. 2009 May; 7(3): 537-68. http://www.elsevier. com/locate/adhoc

15. Schurgers C Srivastava M. Energy Efficient Routing in WirelessSensor Networks. Proceedingsof Military Communications Conference. (MILCOM). 2001. 